\title{
Challenges in Cytomegalovirus (Cmv) Retinitis Management
}

\author{
Denisa Rosati ${ }^{1}$, Sauli Ari Widjaja ${ }^{1,2}$, Ima Yustiarini ${ }^{1,2}$, Randi Montana $^{1,3}$, Wimbo Sasono $^{1,2}$, Muhammad \\ Firmansjah $^{1,2}$, Ady Dwi Prakosa ${ }^{1,2}$, Moestidjab ${ }^{1,2}$, Gatut Suhendro ${ }^{1,2}$
}
${ }^{1}$ Department of Ophthalmology, Faculty of Medicine Universitas Airlangga, Dr. Soetomo General Academic Hospital Surabaya, Indonesia ${ }^{2}$ Vitreoretinal Division, Department of Ophthalmology, Faculty of Medicine Universitas Airlangga, Dr. Soetomo General Academic Hospital Surabaya, Indonesia
${ }^{3}$ Infection Immunology Division, Department of Ophthalmology, Faculty of Medicine Universitas Airlangga, Dr. Soetomo General Academic Hospital Surabaya, Indonesia

\begin{abstract}
Introduction: HIV infection can manifest in a variety of ways in and around the eyes and it is most commonly due to retinal microvasculopathy, neoplasm and also opportunistic infection. Those usually occur associated with a significantly reduced CD4 T-cell counts. In this era of Highly Active Anti Retroviral Therapy (HAART) has caused a major decreasing of the ocular involvement prevalence itself.
\end{abstract}

Case report: A 31 year-old-male came with blurred vision on the right eye, which has started 3 years ago and slowly worsened. Central scotoma also presented previously. Patient was an HIV-AIDS, that placed him on HAART. CD4+ T-lymphocyte count was 3 cells $/ \mathrm{mm}^{3}$. The initial visual acuity was light perception and fundus examination showed Roth spots, massive exudates and hemorrhages covering the optic disc and decreased foveal reflex. Laboratory examination revealed positive Rubella and anti-CMV immunoglobulin$G(I g G)$. He also suffered from lung tuberculosis and took tuberculosis medication regularly. Patient was diagnosed with Cytomegalovirus (CMV) retinitis based on history of illness, fundus examination as well as laboratory testing and given oral induction valganciclovir $900 \mathrm{mg}$ once daily for 3 weeks followed by maintenance dosage.

Result: After valganciclovir induction, there was significant changes with decreased peripapillary exudates, hemorrhages and vasculitis, but the optic disc appeared pale. The patient also had bicytopenia due to valganciclovir therapy that complicate his condition and passed away after 3 months follow up.

Conclusion: CMV retinitis is reported to occur in patient with extreme CD4 count usually less than 50 cells $/ \mathrm{mm}^{3}$. The sooner of proper treatment would likely following better outcome. Making diagnosis of immunosuppresed patient with ocular manifestations was challenging so that comprehensive eye examination in HIV-infected individuals should be conducted. Oral valganciclovir could give satisfactory response to decrease the progression of retinitis but risk of blindness may still occur.

Keywords: cytomegalovirus, CMV retinitis, valganciclovir, HIV-AIDS, CD4+ T-lymphocyte

Cite This Article: ROSATI, denisa. Challenges in Cytomegalovirus (CMV) Retinitis Management. International Journal of Retina, [S.I.], v. 2, n. 2, sep. 2019. ISSN 2614-8536. Available at: https://www.ijretina.com/index.php/ijretina/article/view/91 https://doi.org/10.35479/ijretina.2019.vol002.iss002.91

\section{INTRODUCTION}

*Correspondence to: Denisa Rosati

Department of Ophthalmology, Faculty of Medicine Universitas Airlangga, Dr. Soetomo General Academic Hospital Surabaya, Indonesia

denisarosati88@gmail.com
Human immunodeficiency virus (HIV) remains a major public health problem with 56,000 new HIV infections per year in the United States. ${ }^{1}$ In Indonesia, HIV/AIDS reported case was 3,679 in $2016 .^{2}$ It is estimated that about 70 to $80 \%$ of adult HIV/AIDS patients will experience ocular complication during their illness. ${ }^{3}$ The majority of ocular involvement in HIV is HIV retinopathy and Cytomegalovirus (CMV) retinitis. HIV retinopathy is a non-infectious microvascular disorder characterized by cotton wool spots, microaneurysms, retinal hemorrhages, Roth spots and telangiectatic vascular changes. ${ }^{4}$ 
The most common manifestation of this disorder is cotton wool spots. Unlike infective lesion, cotton wool spot in HIV retinopathy are transient, not visually threatening and tend to disappear within 6-12 weeks. ${ }^{5}$ Retinal hemorrhages are seen less frequently in approximately $30 \%$ of patients with advanced HIV/AIDS. ${ }^{6}$ It may appear as flame-shaped areas when they affect the nerve fiber layer and as dot-and-blot patterns when they affect the deeper layers of the retina. ${ }^{7}$ It can be differentiated from CMV retinitis by the presence of fewer hemorrhages and the absence of subtle iritis or vitritis.

CMV retinitis is the most common ocular opportunistic infection that potentially blinding of patients with HIVAIDS. This disease occurred in up to one-third of HIVinfected patients before the invention of highly active anti retroviral therapy (HAART) and significantly associated with CD4+ T-lymphocyte cell count $<50$ cells $/ \mathrm{mm}^{3}{ }^{8,9}$ The incidence of CMV retinitis in the post-HAART era is estimated to be at most 5.6 cases $/ 100$ persons/year. ${ }^{10} \mathrm{CMV}$ retinitis is characterized by typical white, crumbly areas of retinal necrosis and hemorrhage which is sight threatening if originate in posterior pole. Cotton wool spot is an early manifestation of $\mathrm{CMV}$ retinitis. ${ }^{9}$ The lesions tend to enlarge and coalesce over time, forming large, wedge-shaped areas of involvement. ${ }^{11}$

The clinical forms of CMV retinitis divided as typical form which appear as white spots with many hemorrhages, atypical form which appear as a zone of thinned retina and small dot infiltrate without hemorrhages, perivascular form or "frosted branch angiitis" and optic neuropathy which has the worst prognosis. ${ }^{12}$ Patient with vision disturbance may have irreversible vision loss because of direct damage to the macula and optic nerve, retinal detachment even after CMV retinitis has resolved and immune recovery uveitis. ${ }^{13} \mathrm{CMV}$ retinitis can be treated with ganciclovir or foscarnet, administered systemically or intravitreally. ${ }^{14}$ Another drug of choice is valganciclovir, an orally administered monovalyl ester prodrug of ganciclovir. Induction therapy typically 900 mg once daily for 2-3 weeks followed by maintenance therapy $450 \mathrm{mg}$ once daily. ${ }^{10}$

\section{CASE REPORT}

A 31-year-old man referred to the outpatient clinic with painless visual loss on the right eye since three years ago, started with black dot in the center of his vision, that slowly worsening by time. Patient had been diagnosed with HIV since 4 years and treated with highly active anti retroviral therapy (HAART) such as Emtricitabine/Tenofovir and Lopinavir/Ritonavir. This patient also suffered from lung tuberculosis and had taken anti-tuberculosis fixed-dose combinations (FDCs) for five months. Discrete painless papulo-nodular lesions found over face and neck, assessed as pruritic papular eruption and molluscum contagiosum (Figure 1).

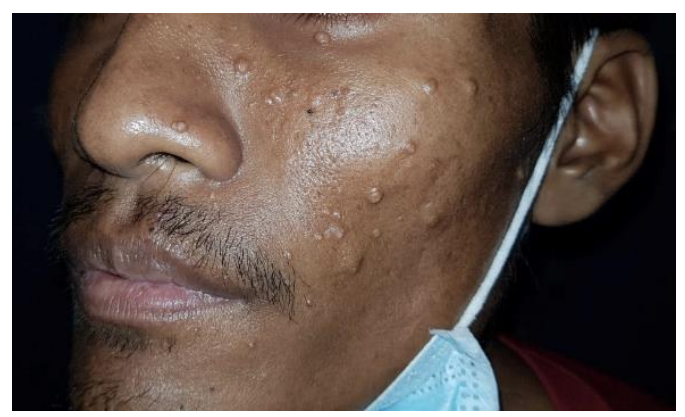

Figure 1. Discrete papulonodular lesions (pruritic papular eruption and moluscum contagiosum)

The visual acuity was light perception on the right eye with mid-dilated pupil, and relative afferent pupillary defect (RAPD) was present at presentation. There was no inflammatory sign on anterior segment. Funduscopy examination revealed massive soft exudates and hemorrhages in posterior pole covering the optic disc, Roth's spot, necrotic lesion due to vasculitis and also reduced foveal reflex (Figure 2). The left eye was within normal limit. Laboratory test showed low CD4 count of 3 cells $/ \mathrm{mm}^{3}$, anemia $8.5 \mathrm{~g} / \mathrm{dl}$, positive IgG rubella and antiCMV. Chest $x$-ray revealed infiltrates with suspicious of lung tuberculosis.

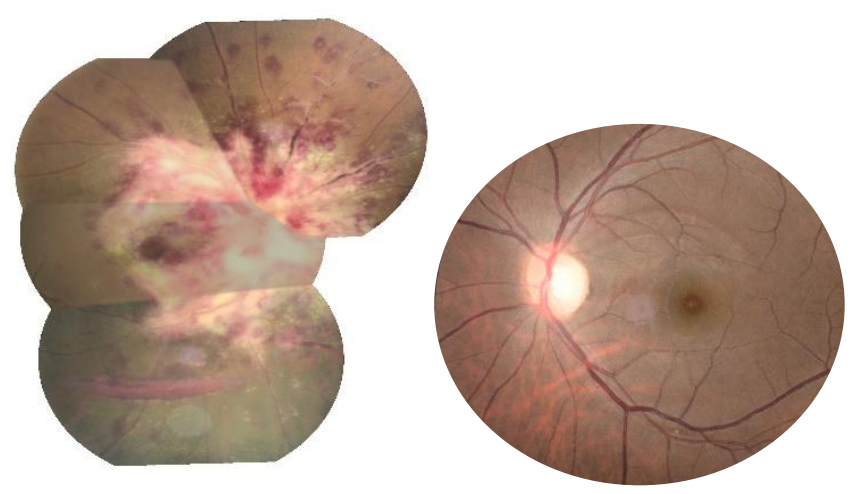

Figure 2. Fundus photograph both eyes before oral valganciclovir therapy. Right eye showed massive soft exudates and hemorrhages in posterior pole

The patient was diagnosed with cytomegalovirus (CMV) retinitis and treated with oral valganciclovir, $900 \mathrm{mg}$ once daily for 3 weeks induction therapy and followed by $450 \mathrm{mg}$ once daily for maintenance therapy. Funduscopy examination was done at the end of induction therapy, showed significant changes with decreased peripapillary exudates, hemorrhages and vasculitis, but the optic disc appeared pale (Figure 3). 
Optical Coherence Tomography (OCT) examination showed macular thickening, intraretinal and subretinal fluid (Figure 4).

Shortly after the end of induction therapy, patient was admitted at Department of Internal Medicine ward due to chronic diarrhea and bicytopenia. Hemoglobin decreased to $6.5 \mathrm{~g} / \mathrm{dl}$ and white blood cells (WBC) count was 1.430 . He had to get PRC transfusion and at that time, considering his weak condition, Internal Medicine Department suggested to postpone valganciclovir maintenance therapy since it appeared to be the underlying cause of his bicytopenia. During hospitalized, visual acuity decreased to no light perception.
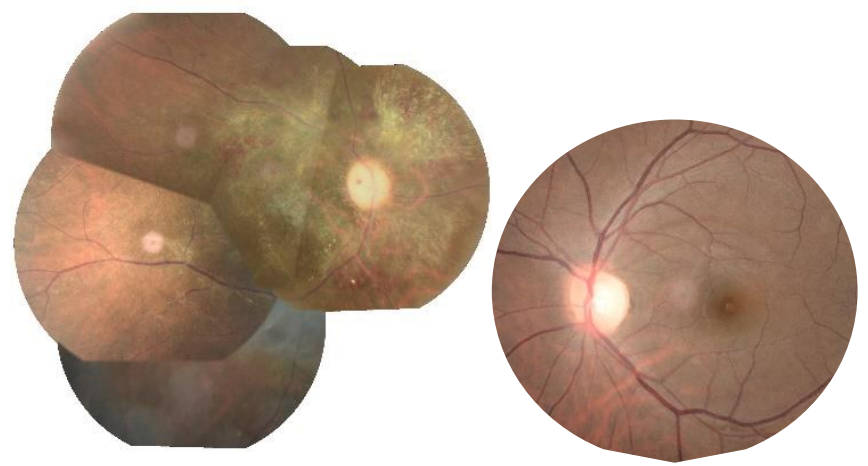

Figure 3. Fundus photograph both eyes after oral valganciclovir induction therapy. Right eye showed significant decreasing of exudates and hemorrhages

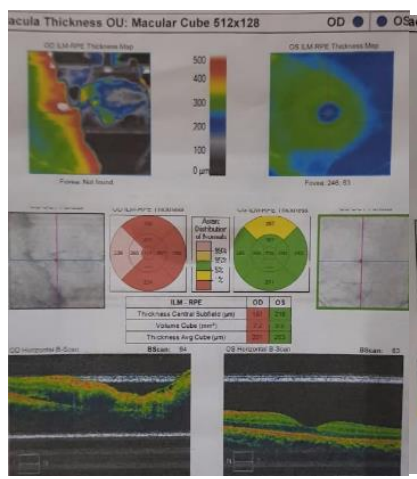

$4 \mathrm{~A}$

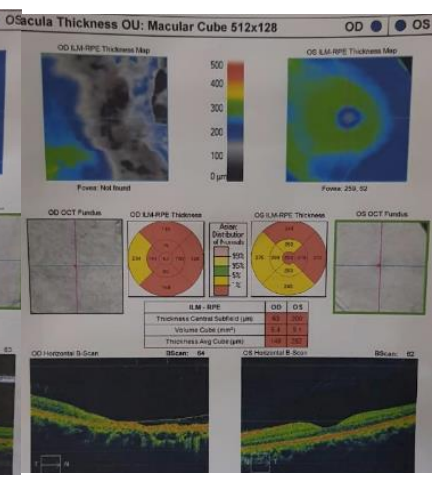

$4 B$
Figure 4. Macular OCT both eyes before valganciclovir induction therapy (4A) and after valganciclovir induction therapy (4B)

One week after, valganciclovir maintenance therapy was continued. During one week follow up, there was no changes in visual acuity, funduscopy showed a pale optic disc, necrotic lesion and decreased exudates with minimal hemorrhages. Patient passed away after 3 months evaluation with remained ocular condition.

\section{DISCUSSION}

HIV/AIDS is undoubtedly a multi systemic disease and ocular involvement occurs in up to $70 \%$ of cases during the natural history of infection. Ocular manifestations of HIVassociated spectrum are very broad and extend from a simple blepharitis to blindness. ${ }^{12}$ The two most common posterior segment ocular manifestations of HIV/AIDS are HIV retinopathy and cytomegalovirus (CMV) retinitis. In this case, the patient complained of gradually painless visual loss with central scotoma and also massive exudates and hemorrhages on funduscopy. HIV retinopathy itself is an occlusive microangiopathy, ${ }^{15}$ which presents as cotton wool spots, microaneurysms and retinal hemorrhages.

However, cotton wool spot associated with HIV retinopathy are usually superficial, smaller lesions that resolve within few months. ${ }^{16}$ Patients with HIV retinopathy rarely have immediate vision loss but there may be damage to the retinal nerve fiber layer, decrease colour vision and also visual field defect. ${ }^{17}$ On the other hand, CMV lesions tend to enlarge and coalesce over time to form larger areas of involvement. Some individuals may complain of blurred vision, scotomas, flashlights or floaters. However, approximately $15 \%$ of infected patients are often asymptomatic despite the presence of extensive or vision threatening CMV retinitis. ${ }^{18}$

CMV retinitis is the most common cause of blindness in patient with HIV-AIDS. The location of infected retina, determine the risk for vision loss. Posterior retinitis threatens the macula and optic nerve and anterior retina increases the risk of retinal detachment. ${ }^{15}$ In this case, the infected retina was posteriorly, which include macula and optic nerve. Hence, the risk of vision threatening was greater. The patient also developed permanent and irreversible visual impairment. Laboratory tests showed a severe decline of $C D 4+$ cell count to only 3 cells/ $\mu \mathrm{L}$. CD4+ cell count of less than 50 cells/ $\mu \mathrm{L}$ of patient with HIV/AIDS is a major risk factor for having active $C M V$ retinitis infection. ${ }^{(19,20)}$ Data from the Longitudinal Study of the Ocular Complications of AIDS (LSOCA) showed that $90 \%$ patients with CMV retinitis had a recent CD4+ cell count of $<50$ cells $/ \mu \mathrm{L}$ and $85 \%$ was using Anti retroviral Therapy (ART) prior to CMV diagnosis. ${ }^{21}$ Conducting routine ophthalmology examinations especially funduscopy on patients presenting for ART initiation with advanced disease is very important. 21,22

Before HAART, treatment of CMV retinitis was a lifelong treatment with specific anti CMV therapy that likely to relapsing of retinitis after two until three weeks of discontinuation. ${ }^{23}$ 
In this HAART era, immune function that is improved by HAART make the cessation of all anti-CMV therapy without reactivation of CMV retinitis possible. ${ }^{24}$ Despite such reports, anti-CMV chemotherapies are still involved especially ganciclovir, foscarnet and cidofovir. ${ }^{25,30}$ In this case, patient was given oral valganciclovir as induction and maintenance therapy. It was the only drug of choice that was available in our center. Historically, the most utilized antiviral agent has been intravenous ganciclovir. In an attempt to make an oral preparation with convenient dosing that has the safety profile, efficacy and bioavailability comparable to ganciclovir, the prodrug was developed. ${ }^{26}$ A randomized control trial study in 2002 showed that valganciclovir, the valine ester of ganciclovir, was found to be as effective as intravenous ganciclovir in more convenient way. In this study, the participants were given oral valganciclovir $900 \mathrm{mg}$ twice daily as induction therapy and the remaining received intravenous ganciclovir $5 \mathrm{mg} / \mathrm{kg}$ for three weeks. ${ }^{27}$ The main adverse effects of both drugs were diarrhea (30\%), neutropenia and anemia $(20 \%){ }^{28}$ This patient also had been admitted to internal medicine ward due to chronic diarrhea and bicytopenia. So that, patient on oral valganciclovir therapy must undergo complete both ophthalmic and systemic evaluations periodically. Depending the medication used, complete blood count, chemistries and intraocular pressure must be checked. Also dilated eye examinations should be performed daily to weekly initially, then 2 weeks after induction therapy. Patients should be undergone CD4 counts and viral load studies. As well. The other reliable treatment for CMV retinitis is intraocular sustained release ganciclovir implants, which have been very effective in treating CMV retinitis but it is not readily available. ${ }^{29}$

In CMV retinitis management, it is often hard to choose the best therapy for the patient considering the side effects. Oral valgancyclovir has been a favourite because it doesn't need IV administration and hospitalization. On the other hand, the cost of the drug is very high, longterm therapy needs strictly good compliance and it causes some toxicities that worsen the condition of immunosuppresed patient. In a point that the patient get remarkable decrease of myelosuppression, sometimes we need to postpone therapy. Consequences, the infection might relapse or remain. It should be avoided if hemoglobin is $<8 \mathrm{~g} \%$, absolute neutrophil count is less than 500 cells $/ \mu \mathrm{L}$, and the platelet count is less than $25,000 / \mu \mathrm{L}^{30}$ In this case, we needed to postpone valganciclovir maintenance therapy due to bicytopenia. Delay of therapy administration bothered the evaluation of medication result. Patient's visual acuity did not improve after 3 weeks maintenance therapy.
Besides, patient got sight-threatening lesions that close to the macula and optic nerve head. The choice of therapy of this condition is injection of $2 \mathrm{mg}$ gancyclovir or $2.4 \mathrm{mg}$ foscarnet. Those medications were not available in our center. On the follow up examination after valganciclovir induction therapy, fundus evaluation showed a remarkable changes. Exudates and hemorrhages were significantly decreased and the optic disc was easier to evaluate. A pale disc with sclerotic vascularities appeared and his visual acuity was no light perception. As optic atrophy had already set in the vision did not improve even though the chorioretinitis patches had resolved. ${ }^{31}$

Visual loss adds to the overwhelming social and economic burden not only for the patient and family itself but also society. We support routine funduscopic examination that has to be included in the standard WHO care package for HIV-infected patients with advanced disease. ${ }^{32}$

\section{CONCLUSION}

Ocular involvement in HIV/AIDS infected patients is very common with broad spectrum of manifestations including non infectious, infectious and neoplasm. CMV retinitis with involvement of posterior pole or owing retinal detachment is the major factor that causes blindness. As CMV retinitis still exists in the HAART era, we need to conduct ophtalmological examinations as part of routine HIV care. Furthermore, standard treatment guidelines for HIV/AIDS patients with CD4+ cell counts $<100$ cells $/ \mu \mathrm{L}$ should include ophtalmological screening.

\section{REFERENCES}

1. Lansky A, Brooks JT, DiNenno E, Heffelfinger J, et.al. Epidemiology of HIV in the United States. JAIDS. J Acquir Immune Defic Syndr 2010. Vol 53: 55-61: S64S68

2. Ministry of Health Republic Indonesia. HIV Epidemiology review Indonesia. 2016-2017. P 26

3. Abu EK, Abokyi S, Yeboah DO, et.al. Retinal Microvasculopathy is common HIV/AIDS patients : A cross sectional study at the cape coast teaching hospital, Ghana. Hindawi Journal of Ophtalmology 2016. Pp 1-5

4. Govender P, Hansraj R, Naidoo KS. Ocular manifestations of HIV/AIDS : A literature review (Part 2). S Afr Optom 2011. Vol 70(2) : 81-88

5. Verma N, Kearney J. Ocular manifestations of AIDS. Pap New Guin Med J 1996. Vol 39 196-199

6. Faia L, Bakri S, DooHo BK, Nader M. HIV. Emedicine website. [Online] Available from: http://www.emedicine. com/OPH/topic417.htm. 2007. Date accessed: 08/2008 
7. Bhatia RS. Ophthalmic manifestations of AIDS. J Ind Acad Clin Med 2002. Vol 3 85-88

8. Sun HY, Peng XY, Li D, Mao FF, You QS, Jonas JB Cytomegalovirus retinitis in patients with AIDS before and after introduction of HAART in China. Eur $J$ Ophthalmol 2014. Vol 24(2):209-215

9. Chen C, Guo CG, Meng L, Yu J, et.al. Comparative analysis of cytomegalovirus retinitis and microvascular retinopathy in patients with acquired immunodeficiency syndrome. J Ophthalmol 2017. Vol $10: 1296-1400$

10. Stewart, Michael. Optimal management of cytomegalovirus retinitis in patients with AIDS. Clin Ophthalmol 2010. Vol 4: 285-299

11. Cheung TW, Teich SA. Cytomegalovirus infection in patients with HIV infection. Mount Sin J Med 1999. Vol 66 113- 124 isE:

12. Chiotan C, Radu L, Serban R, et.al. Posterior segment ocular manifestations of HIV/AIDS patients. J Med Life 2014. Vol 7(3):399-402

13. Heiden D, Ford N, Wilson D, et.al. Cytome- galovirus Retinitis: The neglected disease of the AIDS pandemic. Pub Lib Sci J 2007. Vol 4 1845-1851

14. McCannel C. Cytomegalovirus Retinitis. Retina and Vitreous Section 12 . San Fransisco : American Academy of Ophthalmology. 2016-2017. P.113

15. Jabs DA. Ocular manifestations of HIV infection. Trans Am Ophthalmol Soc 1995. Vol. 93:623

16. Holland GN. AIDS and ophthalmology: The first quarter century. Am J Ophthalmol 2008. Vol. 145(3):397-408

17. Agarwal A, Singh R, Sharma A, et.al. Ocular manifestations in patients with human immunodeficiency virus infection in the pre-HAART versus the HAART era in the North Indian population. Ocular Immunol Inflamm 2016 :396-404

18. Cheung TW, Teich SA. Cytomegalovirus infection in patients with HIV infection. Mount Sin J Med 1999. Vol. $66: 113-124$.

19. Ausayakhun S, Keenan JD, Ausayakhun S. Clinical features of newly diagnosed cytomegalovirus retinitis in northern Thailand. Am J Ophthalmol 2012. Vol. 153(5):923-931.

20. Leenasirimakul $P$, Liu $Y$, Jirawison $C$, et.al. Risk factors for $\mathrm{CMV}$ retinitis among individuals with HIV and low CD4 count in northern Thailand: Importance of access to healthcare. Br J Ophthalmol 2016. Vol. 100:10171021

21. Jabs DA, van Natta ML, Holbrook JT, et.al. Longitudinal study of the ocular complications of AIDS: 1 . Ocular diagnoses at enrollment. Ophthalmology 2007. Vol. 114(4):780- 786

22. Tun N, Smithuis FM, London N, et.al. Mortality in patients with AIDS-related cytomegalovirus retinitis in Myanmar. Clin Infect Dis 2014. Vol. 59(11):1650.

23. Studies of the Ocular Complications of AIDS Research Group in collaboration with the AIDS Clinical Trials Group. Mortality in patients with the acquired immunodeficiency syndrome treated with either foscarnet or ganciclovir for cytomegalovirus. N Engl J Med 1992. Vol..326:213-220

24. Jabs DA, Bolton SG, Dunn JP. Discontinuing anticytomegalovirus therapy in patients with immune reconstitution after combination antiretroviral therapy.Am J Ophthalmol 1998. Vol.126:817-22

25. Spector SA, Weingeist T, Pollard RB, et.al. A randomized, controlled study of intravenous ganciclovir therapy for cytomegalovirus peripheral retinitis in patients with AIDS. J Infect Dis 1993. Vol. 69:557-63

26. Patil J, Sharma A, Kenney C. Valganciclovir in the treatment of cytomegalovirus retinitis in HIV-infected patients. Clin Ophthalmol 2010. Vol 4: 111-119

27. Martin DF, Sierra-Madero J, Walmsley S, et.al. Valganciclovir Study Group. A controlled trial of valganciclovir as induction therapy for cytomegalovirus retinitis. N Engl J Med. 2002. Vol 346(15):1119-1126

28. Liu Y, Chen AS, Kamphaengkham S, et.al. Diagnostic utility of ocular symptoms and vision for cytomegalovirus retinitis. PLOS One 2016. Vol 11(10):e0165564

29. Jung D, Dorr A. Single-dose pharmacokinetics of valganciclovir in HIV and CMVseropositive subjects. $J$ Clin Pharmacol 1999. Vol 39(8):800-804

30. Ho M, Invernizzi A, Zagora S, et al. Presenting Features, Treatment and Clinical Outcomes of Cytomegalovirus Retinitis: Non-HIV Patients Vs HIV Patients. Ocularlmmunology and Inflammation. 2019;29:535542.

31. Musch DC, Martin DF, Gordon JF, et.al. Treatment of cytomegalovirus retinitis with a sustained-release ganciclovir implant. The Ganciclovir Implant Study Group. N Engl J Med. 1997. Vol 337(2):83-90

32. Heiden D, Saranchuk $P$, Tun N, et al. We urge WHO to act on cytomegalovirus retinitis. Lancet Glob Health 2014. Vol. 2(2):e76-e77

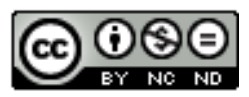

This work licensed under Creative Commons Attribution 